\title{
Focused auditory attention and frequency selectivity
}

\author{
BERTRAM SCHARF, S. QUIGLEY, C. AOKI, N. PEACHEY, and A. REEVES \\ Northeastern University, Boston, Massachusetts
}

\begin{abstract}
The probe-signal method (Greenberg \& Larkin, 1968) was used to determine the percentage of trials in which unpracticed subjects detected (two-interval, forced-choice) a soft, expected sound as compared with an unexpected sound. Pure tones at or near an expected frequency were detected in about $90 \%$ of the trials. Tones more than one-half critical band away were detected near chance ( $50 \%$ ). Complex sounds (a band of noise or a multitone complex) were detected better if they were inside the same critical band as the expected signal than if they were outside the band. A signal that differed spectrally from the expected sound was not detected even though it had the same low pitch, based on a common fundamental frequency. The results may mean that under some conditions focused attention alters sensitivity in the auditory system.
\end{abstract}

A number of experiments have shown that subjects detect a signal light more readily and respond to it more rapidly if it occurs in an expected location than if it occurs in an unexpected location (e.g., Bashinski \& Bacharach, 1980; Posner, 1980; Remington, 1980; van der Heijden \& Eerland, 1973). Moreover, some evidence (Shulman, Wilson, \& Sheehy, 1985) has suggested that the farther the unexpected signal is from the expected locus, the longer the reaction time will be. The usual explanation for these effects is that the observer is able to focus attention on a particular locus so that a stimulus in that region evokes a response more readily. One auditory analog to these visual experiments would be one in which sounds come from different sources in space. Only Posner (1978) appears to have published the results of such auditory measurements. He reported no difference in the reaction time to a sound, whether it came from an expected direction $\left(25^{\circ}\right.$ to one side) or from an unexpected direction. Similarly, our own preliminary results from 6 subjects revealed no difference in the detection of sounds coming from expected as compared with unexpected directions. (However, for some tasks, reaction times to sounds from an expected direction may be shorter.)

Another auditory analog to visual location is sound frequency. In a sense, listening to a sound at a particular frequency is like looking at a light at a particular locus. Just as it is easier to respond to a light in an expected place, it is easier to detect a tone at an expected or "known" frequency (for a recent review, see Swets, 1984). Of the many experimental approaches to this problem, the probesignal method in hearing, developed by Greenberg and

This research was partially supported by NIH Grant R01NS07270. A. Reeves was supported by AFOSR 84-NL-044. We thank Georges Canévet for his help with the program and with the experiments on auditory spatial attention. We appreciate comments about the manuscript by Søren Buus. Requests for reprints should be sent to Bertram Scharf, Auditory Perception Laboratory, Northeastern University, 360 Huntington Ave., Boston, MA 02115.
Larkin (1968), is especially revealing and resembles that used in many of the visual experiments. Indeed, Davis (1981; Davis \& Graham, 1981) adapted the method to study spatial frequency selectivity in vision.

In the probe-signal method, the subject is led to expect, and presumably to listen for a particular sound, the primary. The primary is presented in most trials, but occasionally a different and unexpected sound, the probe, is presented in place of the expected primary. The subject believes that only the primary is presented in every trial and is given no information about the probe. So far, in hearing, the method appears to have been applied only to pure tones varied in sound frequency (Greenberg \& Larkin, 1968; MacMillan \& Schwartz, 1975; Penner, 1972; Yama \& Robinson, 1982). Both the primary and the probe tones were presented at a level near threshold, where they would be heard most of the time when presented alone. The results of such measurements have shown that although the primary is detected on $80 \%$ to $90 \%$ of the trials, a probe distant in frequency is detected at chance level in a two-alternative, forced-choice procedure. However, probes close in frequency to the primary are detected, as is the primary, most of the time. What is close and what is far in frequency seem to be determined by the critical bandwidth, which is roughly $15 \%$ of the center frequency above about $800 \mathrm{~Hz}$ (Scharf, 1970). Probes within approximately half of a critical band on either side of the primary are detected very well, whereas probes more than a critical band away are detected poorly or not at all (Greenberg \& Larkin, 1968; Penner, 1972; Yama \& Robinson, 1982). This suggests that the subject, focusing on a single critical band centered on the primary, hears tones at frequencies within that band and misses those outside. Because the critical band defines the psychoacoustic filter characteristics of the auditory system and plays a vital role in a number of auditory tasks (e.g., detection, loudness, lateralization, and consonance), the probe-signal method would seem to provide a useful means for examining the relation be- 
tween frequency analysis and selective attention in the auditory system.

The present experiments begin to clarify the extent to which poor performance on distant probes stems from the regulation of sensitivity by attention, as opposed to a frequency-dependent criterion that leads subjects to disregard unexpected sounds. They also show that the probesignal method can be used with unpracticed subjects.

\section{GENERAL METHOD}

Experiments 1-9 are divided into two groups. In the five experiments of Group 1, all the stimuli were pure tones; the primary, or expected sound, differed from the probe, or unexpected sound, only in sound frequency. In the four experiments of Group 2, the primary was again a pure tone but the probe was often some other type of sound, such as a band of noise.

Groups 1 and 2 had the same standard sequence of stimuli shown in Figure 1. The duration of the cue was $250 \mathrm{msec}$ and that of the signal (primary or probe) was $350 \mathrm{msec}$. In each trial the cue was presented first, at a low, but clearly audible, level. After a 1-sec interval, two additional intervals, indicated on the subject's terminal screen by the numerals 1 and 2, followed. Each of the latter intervals lasted 350 msec with a 350 -msec interval separating them. One of the two intervals always contained a signal. The subject's task was to press either the 1 or the 2 on the terminal keyboard to indicate in which interval he/she heard the signal. All sounds were presented against a continuous noise that was present throughout a block of trials. Failure to respond by pressing the 1 or 2 within $2 \mathrm{sec}$ was treated as an incorrect response. (Fewer than $4 \%$ of the incorrect responses were such failures.)

All measurements for a single subject were completed in one session that lasted about $1 \mathrm{~h}$. Most sessions began with the measurement of the threshold for the primary signal by a two-interval, forcedchoice, adaptive procedure. Signal level was decreased after two correct responses and increased after one incorrect response, thus converging on the value of $70.7 \%$ correct (Levitt, 1971). Stimuli were

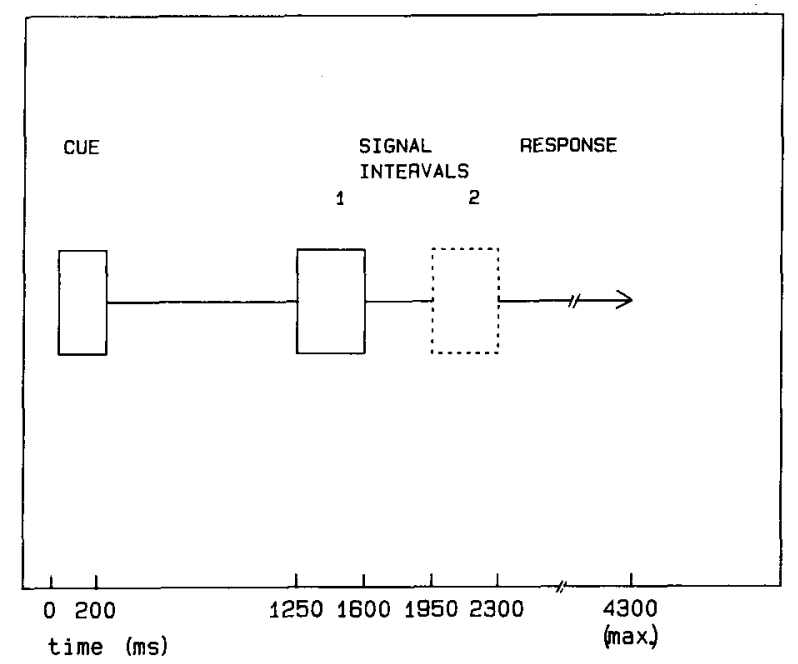

Figure 1. Sequence and time course (in milliseconds) of a single trial. A 250-msec warning cue was followed after $1,000 \mathrm{msec}$ of silence by two intervals marked 1 and 2 . The marked intervals and the separation between them each lasted 350 msec. The signal was presented on a random schedule during one of the intervals. After interval 2, the subjects had to report, within $2,000 \mathrm{msec}$, which interval contained the signal.
Table 1

Sequence and Characteristics of Conditions

\begin{tabular}{llc}
\hline Condition & \multicolumn{1}{c}{ Stimuli } & $\begin{array}{c}\text { Number } \\
\text { of Trials }\end{array}$ \\
\hline 1. Primary-only & $\begin{array}{l}\text { Cue is same as primary in all trials. } \\
\text { Signal is primary in all trials. }\end{array}$ & 104 \\
2. Mixed & $\begin{array}{l}\text { Cue is same as primary in all trials. } \\
\text { Signal is primary in 75\% of trials. } \\
\text { Signal is Probe A in 25\% of trials. }\end{array}$ & 104 \\
3. Mixed & $\begin{array}{l}\text { Cue is same as primary in all trials. } \\
\text { Signal is primary in 75\% of trials. }\end{array}$ & 104 \\
4. Probe-only & $\begin{array}{l}\text { Signal is Probe B in 25\% of trials. } \\
\text { Cue is same as Probe A in all trials. }\end{array}$ & 56 \\
5. Probe-only & $\begin{array}{l}\text { Signal is Probe A in all trials. } \\
\text { Cue is same as Probe B in all trials. }\end{array}$ & 56 \\
\hline Signal is Probe B in all trials. & 56
\end{tabular}

presented in the sequence shown in Figure 1, except that the cue was omitted; also, the subjects were given unlimited response time and feedback on every trial. The measured threshold served as the basis for the levels of the stimuli in the rest of the session.

After the threshold measurements were obtained, which took about $10 \mathrm{~min}$, a standard session, which was divided into the three types of conditions listed in Table 1, took place. In the primary-only condition (Condition 1), which always occurred first, the cue and primary were at the same frequency; the signal was always the primary, and the probe was never presented. In the probe-only conditions (Conditions 4 and 5), which were last, the cue and probe were at the same frequency; the signal was always the probe. In the mixed conditions (Conditions 2 and 3), the cue and primary were at the same frequency, which differed from the probe frequency. The signal was the primary in $75 \%$ of the trials and was the probe in the other $25 \%$. (Their occurrence was quasi-randomized with the restrictions that one of every four consecutive trials contain a probe and that probe trials never immediately follow one another.) Only one probe of a particular frequency or type was included in a given condition. The probe-only conditions provided a measure of the percentage correct for the probe when it was the expected and cued signal; this value served for comparison with that obtained when the probe was unexpected and mixed with the primary.

In all but Experiments 5 and 9, the first three conditions each had 104 trials (the results of the last 100 of which were used), and the last two conditions (Conditions 4 and 5) each had 56 trials, enough to provide a reasonably accurate estimate of the percentage correct. In these experiments, two probes were tested; Probe A was tested before Probe B for about half the subjects and Probe B was tested before Probe A for the remaining subjects. In Experiments 5 and 9 , which tested only one probe, every condition had 104 trials.

\section{Stimuli and Apparatus}

Most of the pure-tone stimuli were generated by programmable oscillators (Krohn-Hite 4141) and then passed through a programmable electronic switch (Colbourn S8404), a programmable attenuator (Charybdis, Model D), and a manual attenuator (HewlettPackard 350D), before being mixed with the background noise and sent through an amplifier (SAE 3100) to a single earphone (Yamaha YM-1). The cue was generally presented $8 \mathrm{~dB}$ above, and the signals $4 \mathrm{~dB}$ above, the threshold for the primary. The background noise was the output of a white-noise generator (Grason-Stadler 455C) filtered (Krohn-Hite 3343) to the appropriate bandwidth for each experiment. The overall level of the noise was usually $60 \mathrm{~dB}$ SPL.

Timing, selection of stimuli, recording of responses, and other experimental details were controlled by a DEC PDP-11/34 
Table 2

Physical Characteristics of Background Noise, Cues, and Signals

\begin{tabular}{|c|c|c|c|c|c|c|}
\hline Experiment & $\begin{array}{c}\text { Noise } \\
\text { Bandwidth }(\mathrm{Hz})\end{array}$ & Cue (Hz) & Primary $(\mathrm{Hz})$ & Probe A $(\mathbf{H z})$ & Probe B (Hz) & $\begin{array}{c}\text { Number } \\
\text { of Subjects }\end{array}$ \\
\hline \multicolumn{7}{|c|}{ Group 1: Pure Tones Only, Frequency Varied } \\
\hline 1 & $500-1500$ & 1000 & 1000 & 925 & 1075 & 10 \\
\hline 2 & $500-1500$ & 1000 & 1000 & 975 & 1025 & 10 \\
\hline 3 & $500-1500$ & - & 1000 & 925 & 1075 & 10 \\
\hline 4 & $300-1800$ & - & 1000 & 600 & 1500 & 9 \\
\hline 5 & $100-4000$ & 400 & 400 & 600 & - & 10 \\
\hline \multicolumn{7}{|c|}{ Group 2: Pure Tones and Complex Sounds } \\
\hline 6 & $500-1500$ & 1000 & 1000 & band of noise & $1075-\mathrm{Hz}$ tone & 9 \\
\hline 7 & $500-1500$ & 1000 & 1000 & band of noise & $1200-\mathrm{Hz}$ tone & 9 \\
\hline 8 & $500-1500$ & 1000 & 1000 & $\begin{array}{l}\text { 2-tone complex } \\
\text { (957 Hz \& } \\
1047 \mathrm{~Hz})\end{array}$ & $\begin{array}{l}\text { 2-tone complex } \\
\text { (1156 Hz \& } \\
1246 \mathrm{~Hz} \text { ) }\end{array}$ & 10 \\
\hline 9 & $100-4000$ & 400 & 400 & $\begin{array}{l}\text { 3-tone complex } \\
(1600 \mathrm{~Hz}, \\
2000 \mathrm{~Hz}, \& \\
2400 \mathrm{~Hz})\end{array}$ & - & 10 \\
\hline
\end{tabular}

minicomputer. The subjects sat in front of a terminal inside a doublewall soundproof room (Eckel Corp.). Table 2 includes the cutoff frequencies for the baokground noise, the characteristics of the cues and signals in each of the nine experiments, and the number of subjects.

\begin{abstract}
Subjects
Data from 87 subjects make up the primary results of the nine experiments. The results were discarded for 16 subjects, whose percentage correct on the primary or probe alone was too low (below $70 \%$ ). The results were incomplete for 17 additional subjects, who did not complete the task because they misunderstood the instructions or because of technical difficulties. Nearly all of the subjects were undergraduates who volunteered to serve in the experiment for extra credit in an introductory psychology course. None was aware of the purpose of the experiments, and few had previously served in any kind of psychology experiment. Each subject served in only one of the nine experiments. No subject reported having a hearing problem.
\end{abstract}

\section{GROUP 1: PURE TONES}

The first five experiments, which had only pure-tone stimuli, are grouped together.

\section{Method}

Experiments 1,2, and 5 followed precisely the standard design shown in Figure 1. Experiments 3 and 4 deviated by omitting the cue in the mixed conditions. Table 2 lists the physical characteristics used in these experiments. The purpose of the experiments was to measure frequency selectivity in an "attention" task with unpracticed subjects and to determine the importance of the cue. Because earlier results with highly practiced subjects (Greenberg \& Larkin, 1968) had shown that detection of a pure-tone probe became poor once its frequency differed from that of the primary by more than about one-half critical band, we set the probe frequencies to 975 and $1025 \mathrm{~Hz}$ (less than one-half critical band away from the primary), to 925 and $1075 \mathrm{~Hz}$ (one-half critical band away), and to 600 and $1500 \mathrm{~Hz}$ (more than a critical band away from the primary). At $1000 \mathrm{~Hz}$, the value of the critical band is approximately $160 \mathrm{~Hz}$ (Scharf, 1970).

\section{Results}

First, we consider the results of Experiments 1, 2, and 4 , which showed the basic effect of frequency selectivity, revealing an "attention band." Experiment 5 extended this demonstration to a lower frequency. We then consider the effect of omitting the cue in Experiments 3 and 4 and the effect of repetition in Experiment 5 and elsewhere.

Attention band. Figure 2 shows the results of Experiments 1,2 , and 4, each of which had a different pair of frequencies, as indicated in Table 2. The results are for all subjects in each experiment. Percentage correct from all trials and subjects in a given condition and experiment are plotted as a function of the signal frequency. Vertical lines represent the $95 \%$-confidence intervals for each data point. (They are omitted for clarity at $1000 \mathrm{~Hz}$, at which the confidence intervals are nearly the same as at the neighboring points.) The unfilled squares represent the primary-only and probe-only conditions in which the cue and signal were always the same, so that the subject was "expecting" a sound at the frequency marked on the abscissa. The three unfilled squares at $1000 \mathrm{~Hz}$ are from Condition 1 (primary only), in which the cue and all signals were at $1000 \mathrm{~Hz}$. This condition was the same for the three groups of subjects, and all three values are close to $90 \%$. Each symbol is based on 900 or 1000 judgments, 100 per subject. The other unfilled squares represent the probe-only conditions, in which the cues and all signals had the same frequencies as the corresponding probes of the mixed conditions. These unfilled squares are very close to $90 \%$, except at $925 \mathrm{~Hz}$. Each value is based on 468 or 520 judgments, 52 per subject. The filled squares represent the unexpected probes presented in $25 \%$ of the trials in the mixed conditions, in which the probe was always different from the cue (if presented).

The results are clear: Unexpected probes near in frequency to the primary and to the cue were detected approximately $85 \%$ of the time-nearly as well as the pri- 


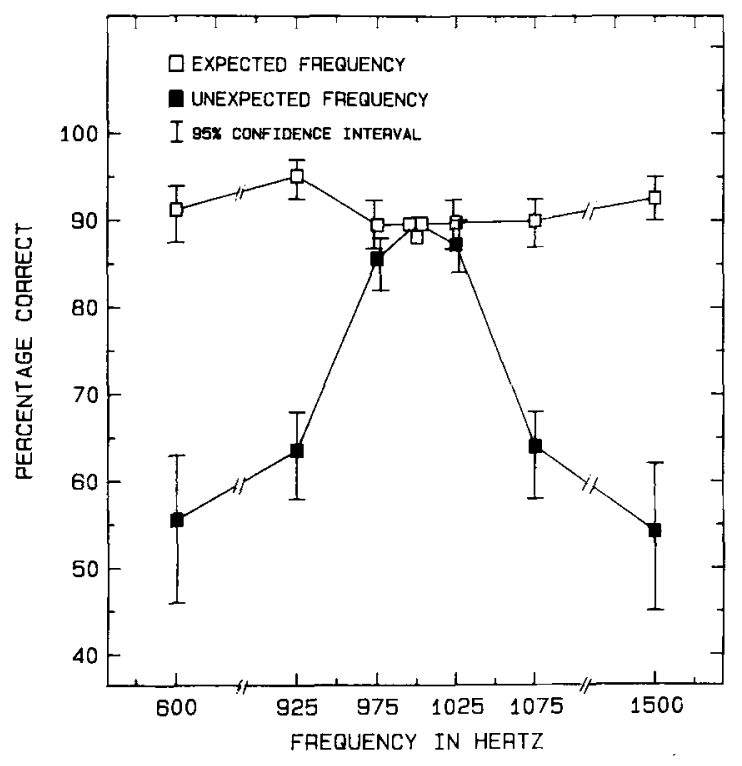

Figure 2. Percentage of trials in which the subject reported correctly the interval that contained the signal. Percentage correct is plotted as a function of the frequency of the pure-tone signal. The unfilled squares represent the trials in which the signal was at the frequency expected by the subject. The filled symbols represent the trials in which the subject expected a signal at $1000 \mathrm{~Hz}$ but received a signal at a different frequency, indicated on the abscissa. If the signal was at the expected frequency, percentage correct hovered around $90 \%$. If the signal was at an unexpected frequency, but close to $1000 \mathrm{~Hz}$ (at 975 and $1025 \mathrm{~Hz}$ ), the percentage correct was greater than $85 \%$. As the unexpected frequency moved away from $1000 \mathrm{~Hz}$, the percentage correct quickly decreased to near $50 \%$, which represents chance performance on this two-interval, forced-choice task. Thus, the unpracticed subjects detected tones at an expected or nearby frequency and missed tones farther away. The confidence interval equals $t \times S / N$, where $t=1.96$ for very large $N$; for small $N, t$ is obtained from the $t$ table with $d f=N-1$ and alpha $=0.05$ (two tails, or alpha $=0.025$, one tail). For example, for $N=11$, $t=2.23$.

mary was detected at the expected frequency. Probes at 925 and $1075 \mathrm{~Hz}$, approximately one-half critical band away from the cue, were detected only $65 \%$ of the time. Probes more than two critical bands away were not detected, inasmuch as the percentages did not differ from chance $(50 \%)$. The filled squares are based on 225 or 250 trials each, which accounts, in part, for the larger confidence intervals. Not shown are the percentages correct for the primary in the mixed conditions (Conditions 2 and 3 ), in which it was presented in three out of four trials. These percentages differed only slightly (by an average of $1 \%$ across the five experiments) from their values when the primary was the only signal (Condition 1). In general, these results are very similar to those of Greenberg and Larkin (1968) for highly trained subjects.

The results for Experiment 5, with the primary at $400 \mathrm{~Hz}$ and the probe at $600 \mathrm{~Hz}$, are similar to those for Experiment 4. When presented alone, the $600-\mathrm{Hz}$ probe was located in the correct interval in $83 \%$ of the trials; as a probe, it was correctly located in $50 \%$ of the trialschance performance. Thus, the effect of frequency selec- tivity measured by the probe-signal method is as clear at $400 \mathrm{~Hz}$ as it is at $1000 \mathrm{~Hz}^{1}$

Effect of cue. In Experiment 3, even with no cues in the mixed conditions, detection of the unexpected probes at 925 and $1075 \mathrm{~Hz}$ - on the edge of the critical band surrounding the $1000-\mathrm{Hz}$ primary - was down to $79 \%$ from $94 \%$ in the probes-only conditions. Nevertheless, $79 \%$ detection was better than $64 \%$ for the same unexpected probes in Experiment 2, in which there was a cue. On the other hand, omitting the cue in Experiment 4 had little effect on the detection of probes that were at frequencies far from the primary, at 600 and $1500 \mathrm{~Hz}$. Thus, the effect of the cue depends, at least in part, on how far the probe lies from the primary.

Effect of repetition. The results show that the primary was not detected better than the probe simply because the primary was presented much more frequently, both as cue and as signal. In Experiment 5, we repeated the mixed condition after having run the probe-only condition, with the cues and all signals set to the probe frequency. Thus, at the start of Condition 4, which was a repetition of Condition 2 (mixed), the subject had just been exposed to 104 trials on which he/she heard only the probe frequency. Yet, the percent correct on the probe trials in the replication was only 51.5 , not significantly different from chance or from the $49.9 \%$ performance in Condition 2 . Furthermore, in Experiment 4 reducing the exposure to the primary by removing the cue in the mixed conditions did not prevent chance performance in the probe trials.

\section{Discussion}

The results are consistent with a single-band model of selective attention according to which the subject attends to a narrow range of frequencies. Under the conditions of the present group of experiments, this range appears to be approximately the same as the critical band. Not only does the detection of the signal fall off when the frequency is more than a half critical band away from the primary, but the increase in detection of the probe in Experiment 3 , in which the cue was eliminated, is easily understood on the basis of a single-band model. With the elimination of the cue at $1000 \mathrm{~Hz}$, the center of the listening band might well have drifted at times from the primary frequency to a frequency intermediate between the primary at $1000 \mathrm{~Hz}$ and the probe at $1075 \mathrm{~Hz}$. In that position, both the primary and the probe would fall within the attention band, thereby permitting detection of either. An analysis of individual data also suggests the possibility that for some subjects the center of the band shifted to a new, intermediate locus, where it remained throughout the mixed conditions (Conditions 2 and 3 ). Six of the 10 subjects detected the $925-\mathrm{Hz}$ tone as well when it was the probe as when it was presented alone. Likewise, 4 subjects detected the $1075-\mathrm{Hz}$ tone equally well whether it was the probe or not. These subjects' average change in the percentage correct from probe trials (Conditions 2 and 3) to probe-only trials (Conditions 4 and 5) was $1 \%$. For the rest of the subjects, the average change was 
$30 \%$. The possibility that some subjects responded to the absence of the cue by shifting their attention band but others did not is interesting but rather conjectural, because it is based on the results from only 10 , unpracticed subjects. Nevertheless, the results of Experiment 4 support the shifting-band explanation. In this experiment there was also no cue, but the probes were several critical bands away from the primary so that a single critical band could not encompass both primary and probe, as was possible in Experiment 3. The outcome was unequivocal: in all but one of the 20 cases (10 subjects $\times 2$ probes), detection of a probe in mixed trials was well below that in probe-only trials, and the averages for the probes that were unexpected were near chance.

Nonetheless, a single-band model cannot account for the data of a number of other experiments. The applicable model may depend on the filtering strategies that subjects adopt to meet the demands of a particular situation and experiment (cf. Swets, 1963). Buus, Schorer, Florentine, and Zwicker (1986) have proposed that the general strategy is a nonoptimal multiband one that reduces, in effect, to a single-band strategy in conditions like those of the present experiments. According to Buus et al., in a version of the probe-signal paradigm such as ours, listeners do not use information in bands other than the one surrounding the primary. In other versions of the paradigm, pairs of cues have led listeners to expect two primaries, which they detect equally well although the primaries are far apart in frequency, while they miss probes falling between the primaries (MacMillan \& Schwartz, 1975; Johnson \& Hafter, 1980). Monetary payoffs can also lead subjects to adopt either a single- or a multiband strategy in experiments employing the probe-signal method (Penner, 1972). Thus, clearly, subjects can listen to one, or to more than one, band at a time, although how they come to choose their listening strategies, for example, whether by control or automatic processing (Schneider, Dumais, \& Shiffrin, 1984), is not known. In any case, the usual assumption is that the information in the bands containing undetected probes does not reach the decision mechanism.

A different explanation for poor detection of the probes assumes (even for our two-interval, forced-choice procedure) that the subjects are aware of the probes-that, indeed, they hear them but do not heed them. In other words, the listeners are just as sensitive to the probe as to the primary, but they disregard it, perhaps because they adopt less than optimal criteria for unexpected frequencies. A probe might also be disregarded if the subjects hear it but miscode it, treating it as part of the noise. In signal-detection terms, this means a poor choice of decision axis, rather than a poor choice of criterion on a given axis. Accordingly, the choice of which listening interval to report might depend not only on the magnitude of sensory events, but also on their quality. Whatever its basis, the heard-but-not-heeded hypothesis needs to be tested more directly; that is the purpose of the experiments of Group 2.

\section{GROUP 2: COMPLEX SOUNDS}

The four experiments of Group 2 were designed to determine to what extent, if any, subjects heard probe signals but ignored them if they were too different from the primary. According to this hypothesis, probes close in spectral content to the primary but different in other ways also should be disregarded. According to the single-band hypothesis, such probes-even when differing in other respects-should be detected, because they fall in the same listening or attention band as the primary. In Experiments 6 and 7 , we attempted to test this explanation by using, as the probe, a narrow band of noise centered on the frequency of the primary. In Experiment 8, we used, instead of a band of noise, a two-tone complex also centered on the primary. In each of these cases, the probe, a complex sound, and the primary, a pure tone, sounded quite different but fell within the same critical band. The converse was true in Experiment 9, in which probe and primary were far apart in frequency but had the same fundamental frequency and periodicity pitch.

\section{Method}

Table 2 provides information about each of the four experiments of Group 2. In Experiments 6 and 7, Probe A was a narrow band of noise with sharp skirts at the cutoff frequencies of 925 and $1075 \mathrm{~Hz}$. Probe B was a pure tone at $1075 \mathrm{~Hz}$. The condition with the noise probe was always run before the condition with the puretone probe. Experiment 6 was like all the preceding experiments in that no feedback was given; in Experiment 7 feedback was given in every trial.

In Experiment 8, the probes were complex sounds composed of two pure tones $90 \mathrm{~Hz}$ apart and centered either on $1000 \mathrm{~Hz}$ or on $1200 \mathrm{~Hz}$. In Experiment 9, the probe was a three-tone complex made up of frequencies at 1600,2000 , and $2400 \mathrm{~Hz}$. The complex was produced by mixing the outputs of three manually controlled oscillators (Hewlett-Packard 241A).

The subjects came from the same undergraduate pool as in the experiments of Group 1. Of the 87 subjects described above, 38 served in the experiments of Group 2.

\section{Results and Discussion}

Table 3 presents the overall percentages of correct responses for Experiments 6 through 9. For each experiment, the class (primary or probe) and the type of signal are listed. The fourth column lists the percentage correct for the primary and probe signals when presented alone (i.e., cue and all signals the same). The last column lists the percentages correct for conditions in which primary and probe were intermixed. (Since in all but Experiment 9 there were two blocks of trials with different probes, the percentage correct for the primary intermixed with the probe was taken as the average over both blocks.) In all four experiments, intermixing probe signals with primary signals caused a greater reduction in the percentage correct for probes far in frequency from the primary than for those near in frequency. This finding is inconsistent with the heard-but-not-heeded model because the near probe, a complex sound (multitone complex or band of 
Table 3

Percentages Correct (\%) of Responses to Each Class of Signal in Experiments 6, 7, 8, and 9

\begin{tabular}{|c|c|c|c|c|}
\hline \multirow[b]{3}{*}{ Experiment } & \multirow{2}{*}{\multicolumn{2}{|c|}{ Signal }} & \multicolumn{2}{|c|}{ Condition } \\
\hline & & & \multirow{2}{*}{$\begin{array}{c}\text { Primary- or } \\
\text { Probe-Only (\%) }\end{array}$} & \multirow[b]{2}{*}{ Mixed (\%) } \\
\hline & Class & Type & & \\
\hline \multirow[t]{3}{*}{6} & Primary & $1000-\mathrm{Hz}$ tone & 92 & 86 \\
\hline & Probe A & $\begin{array}{l}\text { Band of noise } \\
\text { at } 1000 \mathrm{~Hz}\end{array}$ & 85 & 74 \\
\hline & Probe $\mathrm{B}$ & $1075-\mathrm{Hz}$ tone & 88 & 69 \\
\hline \multirow[t]{3}{*}{7} & Primary & $1000-\mathrm{Hz}$ tone & 88 & 90 \\
\hline & Probe A & $\begin{array}{c}\text { Band of noise } \\
\text { at } 1000 \mathrm{~Hz}\end{array}$ & 92 & 79 \\
\hline & Probe B & $1200-\mathrm{Hz}$ tone & 90 & 51 \\
\hline \multirow[t]{3}{*}{8} & Primary & $1000-\mathrm{Hz}$ tone & 90 & 84 \\
\hline & Probe A & $\begin{array}{l}\text { 2-tone complex } \\
\text { at } 1000 \mathrm{~Hz}\end{array}$ & 78 & 69 \\
\hline & Probe B & $\begin{array}{l}\text { 2-tone complex } \\
\text { at } 1200 \mathrm{~Hz}\end{array}$ & 79 & 55 \\
\hline \multirow[t]{3}{*}{9} & Primary & $400-\mathrm{Hz}$ tone & 91 & 90 \\
\hline & Probe & $\begin{array}{l}\text { 3-tone complex } \\
\text { at } 2000 \mathrm{~Hz}\end{array}$ & 87 & 51 \\
\hline & $\begin{array}{l}\text { Probe } \\
\text { (replication) }\end{array}$ & 3-tone complex & - & 49 \\
\hline
\end{tabular}

noise), and the primary, a pure tone, sounded quite different from each other. However, the results are not entirely consistent with an attention-band hypothesis because the near probes were not detected as well when unexpected as when expected, or as well as the primaries with which they shared the same critical band. ${ }^{2}$

Because the narrow-band noise in Experiment 6 was detected less well when it was probe than when it was primary, we introduced feedback in Experiment 7. Were the decline in detection an example of hearing but not heeding, then feedback should have improved performance by permitting the subject to learn to treat the qualitatively different probe as signal and not as noise. However, there was no improvement. In Experiment 6, in which no feedback was given, accuracy declined $11 \%$ when the noise signal was unexpected and in Experiment 7 , in which feedback was given, it declined $13 \%$. (Feedback was also of no help in the detection of the $1200-\mathrm{Hz}$ tone, which was at chance when unexpected.) The failure of feedback to help speaks against a criterion effect, although the possibility remains that the number of trials was too small to give feedback a fair chance. Among alternative explanations for the poorer detection of the unexpected noise would be instability of the attention filter or critical band. Assume that the filter does not remain fixed on $1000 \mathrm{~Hz}$ but moves about. If the instability does not exceed \pm 0.5 critical bands, detection of a pure tone at $1000 \mathrm{~Hz}$ would be essentially unaffected, but detection of a narrow-band noise would suffer because part of the noise would sometimes fall outside the attention band and the signal-to-noise ratio would worsen.

In Experiment 9, the three-tone complex was located far from the frequency of the primary; although its fundamental frequency (and periodicity pitch) was the same as that of the primary, the percentage correct declined by $36 \%$ to chance. The subjects did not detect the probe despite its having a periodicity pitch equal to the puretone pitch of the primary. In a separate experiment in our laboratory, Huanping Dai obtained a similar outcome for a probe at just double the frequency of the primary (unpublished). Despite the qualitative similarity between tones an octave apart (Scharf \& Houtsma, 1986), the subjects did no better in detecting a probe at $2000 \mathrm{~Hz}$ than in detecting one at $1500 \mathrm{~Hz}$ when they were expecting a 1000-Hz primary; detection of both probes (presented in separate conditions) was near chance. Thus, when frequencies are far apart, a single, common characteristic, such as periodicity pitch or octave similarity, does not overcome the effects of frequency selectivity.

Another way to test the hypothesis that subjects ignore soft, unexpected signals is to tell them beforehand that sometimes a sound other than the cue will be presented. Accordingly, we ran two additional experiments. The first was a replication of Experiment 1, except that the subjects were told at the beginning of Condition 2 that a lower (or higher) pitch tone would be presented on some trials. The probes were set at frequencies one-half critical band from the primary at 925 and $1075 \mathrm{~Hz}$. These subjects detected the near frequencies better than did earlier subjects (Experiments 1 and 7) who had not expected signals other than the primary. Percentages correct were near $75 \%$ on the probes in the mixed conditions, approximately $10 \%$ higher than they were in Experiment 1. However, they were significantly below the $88 \%$ correct for the probe presented alone. This result is equivocal because the subjects could have shifted their critical band to encompass both primary and probe, just as some subjects had seemed to do in Experiment 3 when the cue was removed. Therefore, we ran 
a second additional experiment with frequencies more than a critical band away, at 700 and $1200 \mathrm{~Hz}$, so that subjects could not shift their listening band to encompass both primary and probe simultaneously. The percentage correct on the probes dropped to $60 \%$, close to chance but still somewhat better than in Experiment 7, in which the $1200-\mathrm{Hz}$ tone was detected at chance level $(51 \%)$.

\section{GENERAL DISCUSSION}

The main finding is clear: naive, untrained subjects quickly show strong frequency selectivity in listening for pure tones. Accordingly, extensive training is not needed to study selective attention, using the probe-signal procedure. More important, this finding emphasizes the naturalness and rapidity with which listeners fine-tune their listening. Just how they do it remains uncertain. We will briefly review two contending, but not mutually exclusive, explanations. The heard-but-not-heeded hypothesis emphasizes the response side, whereas the single-band hypothesis emphasizes the sensory input.

The heard-but-not-heeded hypothesis assumes that subjects make a conscious (heard) rejection (not heeded) of probe signals. They believe they are thereby avoiding false alarms. Graham, Kramer, and Haber (1985) grappled with the same problem in vision. Their objective results on the detection of gratings that were varied in spatial frequency seemed to support "conscious rejection of false alarms," that is, of gratings at frequencies sufficiently different from the expected frequency. However, subjects rarely reported making conscious rejections, even when tested by a version of the probe-signal method (Davis \& Graham, 1981).

Graham et al. (1985) also presented anecdotal evidence. Experimenters, when setting their stimuli, often fail to see a grating at an unexpected frequency even at contrasts "far above threshold." At some still higher contrast, the grating is suddenly seen, after which its contrast can be reduced to "normal" values. We had similar experiences when developing and testing our own experiments. After a change in the warning cue and signal, we often had difficulty hearing either one on the first few trials. One of our naive subjects heard neither one throughout a probealone block, but heard them easily after a brief demonstration.

Further evidence against the poststimulus, responsechoice hypothesis comes from the failure of instructions to improve performance on the probe trials. Telling subjects that signals other than the primary would be presented did not help them to detect distant probes. Similarly, when experimenters ran themselves, knowing exactly what stimuli were being presented, they still did poorly on probe trials.

We conclude that a conscious, response-choice explanation such as the heard-but-not-heeded hypothesis probably plays only a small role in the probe-signal procedure. We suggest that the subject is able to make a choice among sensory events on the basis of special criteria, and readies the "filter" prior to stimulation so as to facilitate reception of relevant signals. This facilitation may even influence filtering in the cochlea, which implies finetuning in the sensory periphery. That a preparatory set can result in exquisite control at the body's periphery is evident in the readying of particular muscles for coordinated movement (Requin, 1980). The careful listener focuses attention on a particular frequency region. In our experiments, the choice of the region was usually determined by the cue; in other experiments, it was determined by experience with samples of the primary (Greenberg \& Larkin, 1968; MacMillan \& Schwartz, 1975; Penner, 1972). The filter, however, can also be set by anticipating the frequency region that will encompass a signal, on the basis of its occurrence in an ascending or descending frequency series (Howard, O'Toole, Parasuraman, \& Bennett, 1984; Okita, 1979).

Having opted for the sensory-side, single-band hypothesis, we are left with the question of how the filtering is achieved. One possibility is centrifugal control via the olivary-cochlear bundle, which innervates most of the cochlear hair cells. We postulate an active control process that produces an improved signal-to-noise ratio in the region of the cochlea that subserves the frequencies to be detected. A number of physiological experiments have shown that the olivary-cochlear bundle can suppress excitation from the cochlea (e.g., Wiederhold, 1970). Electrophysiological evidence shows that in cats (Oatman \& Anderson, 1977) and in humans (Lukas, 1980, 1981) attention to a visual task reduces and delays the neural potentials evoked by sound in the cochlear nucleus and inferior colliculus. In humans, attention to a targeted auditory locus has been shown to enhance evoked potentials from the periphery of the auditory nervous system (McCallum, Curry, Cooper, Pocock, \& Papakostopoulos, 1983). These results revive the possibility that efferent input under attentional control may attenuate the responsiveness of the auditory system to sound. It remains to be shown that efferent input can achieve the fine-tuning in the cochlea required by our psychoacoustical data.

Another possibility is that the response of the cochlea is wholly independent of the listener's expectations and intentions, and that only at some higher level does focused attention lay the basis for differential responding to primary and probe. For example, if a signal at the primary frequency is expected, the auditory system might weight that part of the neural input more heavily than other parts, resulting in greater sensory magnitude (Buus et al., 1986; Green, 1958). This hypothesis places the weighting at a preconscious level, unlike the heard-but-not-heeded hypothesis. Although it is impossible to specify a neural site for such a weighting, the failure of similarity in periodicity pitch to improve detection of the probe suggests a more peripheral level because periodicity pitch can be formed dichotically (see Scharf \& Houtsma, 1986). Given a peripheral site, the weighting may be related to the processing negativity seen in evoked responses to attended auditory input (Näätänen, 1982).

From the results of these experiments, we can reach the following conclusions. Unpracticed subjects-like practiced ones-detect a weak, expected sound more read- 
ily than a weak, unexpected sound. When the sounds are pure tones, the disadvantage in detection is greatest for unexpected tones more than a half critical band distant from the expected tone.

In support of the attention-band hypothesis, unexpected sounds that are not pure tones are detected better when they fall in the same critical band as the expected sound. However, they are not detected quite as well as similarly placed pure tones, perhaps due to greater qualitative differences. This result supports the notion that unexpected sounds are occasionally heard but not heeded because they are confused with the background noise.

The results suggest that in this version of the probesignal paradigm, subjects use a single-band listening strategy. Changes in instructions or in the stimulus sequence (e.g., elimination of a warning cue) may lead some subjects to shift the locus of their listening band (which seems to be the same as the attention band and the critical band) to encompass both primary and probe signals; as a result, they detect both signals equally well.

Although qualitative differences between expected and unexpected signals may on occasion lead a subject to ignore an unexpected signal (as noted above), the major determinant of the expected signal's advantage appears to be its locus in the attended critical band. Thus, an unexpected high-frequency complex with the same low (residue or periodicity) pitch as an expected pure tone is not detected despite the similarity in pitch.

Finally, could it be that attending to a particular frequency region leads to enhanced signal reception in that region at the cochlear level and/or reduced reception in other frequency regions?

\section{REFERENCES}

BASHinsti, H. S., \& BACHARACH, V. R. (1980). Enhancement of perceptual sensitivity as the result of selectively attending to spatial locations. Perception \& Psychophysics, 28, 241-248.

BuUs, S., Schorer, E., Florentine, M., ZWicker, E. (1986). Decision rules in detection of simple and complex tones. Journal of the Acoustical Society of America, 80, 1646-1657.

DAVIS, E. T. (1981). Allocation of attention: Uncertainty effects when monitoring one or two visual gratings of noncontiguous spatial frequencies. Perception \& Psychophysics, 29, 618-622.

DAVIS, E. T., \& GRAHAM, N. (1981). Spatial frequency uncertainty effects in the detection of sinusoidal gratings. Vision Research, 21, 705-712.

Graham, N., Kramer, P., \& Haber, N. (1985). Attending to the spatial frequency and spatial position of near-threshold visual patterns. In M. I. Posner \& O. S. Marin (Eds.), Attention \& Performance XI (pp. 269-284). Hillsdale, NJ: Erlbaum.

GREEN, D. M. (1958). Detection of multiple component signals in noise. Journal of the Acoustical Society of America, 30, 904-911.

Greenberg, G. Z., \& LARKIN, W. D. (1968). Frequency-response characteristic of auditory observers detecting signals of a single frequency in noise: The probe-signal method. Journal of the Acoustical Society of America, 44, 1513-1523.

Howard, J. H., JR., O'Toole, A. J., Parasuraman, R., \& Bennett, K. B. (1984). Pattern-directed attention in uncertain-frequency detection. Perception \& Psychophysics, 35, 256-264.
Johnson, D. M., HafTer, E. R. (1980). Uncertain-frequency detection: Cueing and condition of observation. Perception \& Psychophysics, 28, 143-149.

LEVITT, H. (1971). Transformed up-down methods in psychoacoustics. Journal of the Acoustical Society of America, 49, 467-477.

LUKAS, J. H. (1980). Human auditory attention: The olivocochlear bundle may function as a peripheral filter. Psychophysiology, 17, 444-452.

LuKas, J. H. (1981). The role of efferent inhibition in human auditory attention: An examination of the auditory brainstem potentials. Intermational Journal of Neuroscience, 12, 137-145.

MacMullan, N. A., SCHWARTz, M. (1975). A probe-signal investigation of uncertain-frequency detection. Joumal of the Acoustical Society of America, 58, 1051-1058.

McCallum, W. C., Curry, S. H., Cooper, R., Pocock, P. V., \& Papakostopoulos, D. (1983). Brain event-related potentials as indicators of early selective processes in auditory target localization. Psychophysiology, 20, 1-17.

NÄÄTÄNEN, R. (1982). Processing negativity: An evoked-potential reflection of selective attention. Psychological Bulletin, 92, 605-640.

Oatman, L. C., \& ANDERson, B. W. (1977). Effects of visual attention on tone burst evoked auditory potentials. Experimental Neurology, 57, 200-211.

OkITA, T. (1979). Event-related potentials and selective attention to auditory stimuli varying in pitch and localization. Biological Psychology, 9, 271-284.

Penner, M. J. (1972). The effect of pay-offs and cue tones on detection of sinusoids of uncertain frequency. Perception \& Psychophysics, 11, 198-202.

PoSNER, M. I. (1978). Chronometric explorations of mind. Hillsdale, NJ: Erlbaum.

PoSNER, M. I. (1980). Orienting of attention. Quarterly Journal of Experimental Psychology, 32, 3-25.

REMINGTON, R. W. (1980). Attention and saccadic eye movements. Journal of Experimental Psychology: Human Perception \& Performance, 6, 726-744.

ReQuiN, J. (1980). Toward a psychobiology of preparation for action. In G. E. Stelmach \& J. Requin (Eds.), Tutorials in motor behavior (pp. 373-398). Amsterdam: North-Holland.

SCHARF, B. (1970). Critical bands. In J. V. Tobias (Ed.), Foundations of modern auditory theory (Vol. 1, pp. 157-202). New York: Academic Press.

SCHARF, B., \& Houtsma, A. (1986). Audition II. In K. Boff, L. Kaufman, \& J. Thomas (Eds.), Handbook of perception \& human performance (Vol. 1, pp. 15.1-15.60). New York: Wiley.

SChNeIDER, W., DUMAIS, S. T., \& ShIFrRIN, R. M. (1984). Automatic and control processing and attention. In R. Parasuraman \& D. R. Davies (Eds.), Varieties of attention (pp. 1-27). New York: Academic Press.

Shulman, G. L., Wilson, J., \& Sheehy, J. B. (1985). Spatial determinants of the distribution of attention. Perception \& Psychophysics, 37, 59-65.

SwETS, J. A. (1963). Central factors in auditory frequency selectivity. Psychological Bulletin, 60, 429-440.

SweTs, J. A. (1984). Mathematical models of attention. In R. Parasuraman \& D. R. Davies (Eds.), Varieties of attention (pp. 183-242). New York: Academic Press.

van der Heuden, A. H. C., Eerland, E. (1973). The effects of cueing in a visual signal detection task. Quarterly Journal of Experimental Psychology, 25, 496-503.

WIEDERHOLD, M. L. (1970). Variations in the effects of electric stimulation of the crossed olivocochlear bundle on cat single auditory-nervefiber responses to tone bursts. Joumal of the Acoustical Society of America, 48, 966-977.

YAmA, M. F., Robinson, D. E. (1982). Comparison of frequency selectivity for monaural and binaural hearing systems: Evidence for a probe-signal frequency procedure. Journal of the Acoustical Society of America, 71, 694-700. 


\section{NOTES}

1. Experiment 5 differed from the earlier experiments not only in primary frequency but also in method. No threshold measurements were made; instead, the results of 10 other subjects, who participated in a separate experiment for threshold only, were used to determine the reference for the sensation levels. Masked thresholds of these subjects were all close to the mean $(39.5 \mathrm{~dB}$ at $400 \mathrm{~Hz}$ and $40.0 \mathrm{~dB}$ at $600 \mathrm{~Hz})$; the standard deviations were less than $1 \mathrm{~dB}$. Accordingly, the primary and the probe were set to $43.5 \mathrm{~dB}$, nearly $4 \mathrm{~dB}$ above the measured threshold. Detection of the primary remained high; the mean percentage correct on the primary in Condition 1 was equal to $87 \%$. Moreover, because Experiment 5 had only one probe, only a single probe-only condition was run, immediately following Condition 2 , the mixed condition. The same mixed condition was repeated at the end.

2. A few words need to be said about the absolute values of the percentages in Experiment 8. The percentages for the complexes presented alone were only 78 and 79 , considerably less than the values in other experiments, in which probes were usually detected about $90 \%$ of the time when presented alone. Measured by the adaptive procedure, the thresholds for the complexes were only $0.6 \mathrm{~dB}$ higher than that for the 1000-Hz primary, and so the complexes should also have been detected close to $90 \%$ of the time. We have no explanation for the lower percentages of detection, but it suggests that the psychometric functions are shallower for the two-tone complexes than for the single tone. We encountered a similar phenomenon in Experiments 6 and 7, in which the level of the band of noise had to be set 2 to $3 \mathrm{~dB}$ higher than that of the $1000-\mathrm{Hz}$ tone to be detected near $90 \%$ in the probe-alone blocks.

(Manuscript received April 23, 1986; revision accepted for publication March 27, 1987.) 